CNS Spectrums (2016), 21, 466-477. C Cambridge University Press 2016. This is an Open Access article, distributed under the terms of the Creative Commons Attribution licence (http://creativecommons.org/licenses/by/4.0/), which permits unrestricted re-use, distribution, and reproduction in any medium, provided the original work is properly cited.

\title{
Once-monthly paliperidone palmitate compared with conventional and atypical daily oral antipsychotic treatment in patients with schizophrenia
}

\author{
Edward Kim, ${ }^{*}$ Christoph U. Correll, ${ }^{2,3}$ Lian Mao, ${ }^{4}$ H. Lynn Starr, ${ }^{\prime}$ and \\ Larry Alphs'
}

\footnotetext{
${ }^{1}$ Janssen Scientific Affairs, LLC, Titusville, New Jersey, USA

${ }^{2}$ Hofstra Northwell School of Medicine, Hempstead, New York, USA

${ }^{3}$ Department of Psychiatry, The Zucker Hillside Hospital, Glen Oaks, New York, USA

${ }^{4}$ Janssen Research \& Development, LLC, Titusville, New Jersey, USA
}

Objective. This analysis of the Paliperidone Palmitate Research in Demonstrating Effectiveness (PRIDE) study (NCT01157351) compared outcomes after administration of once-monthly paliperidone palmitate (PP) vs conventional oral antipsychotics (COAs) or atypical oral antipsychotics (AOAs).

Methods. PRIDE was a 15-month study of 444 individuals with schizophrenia and a history of incarceration. They were randomly assigned to PP or to 1 of 7 commonly prescribed OAs. Primary endpoint was time to first treatment failure (TF). Event-free probabilities were estimated using the Kaplan-Meier method; treatment group differences (PP vs COAs, PP vs AOAs, and $\mathrm{PP}$ vs oral paliperidone/risperidone) were assessed using a log-rank test. Hazard ratios (HRs) and 95\% confidence intervals (CIs) were estimated using Cox proportional hazards regression models. No adjustment was made for multiplicity.

Results. Compared with PP, risk for first TF was 34\% higher with COAs (HR: 1.34; 95\% CI: 0.80-2.25), 41\% higher with AOAs (HR: 1.41; 95\% CI: 1.06-1.88), and 39\% higher with paliperidone/risperidone (HR: 1.39; $95 \%$ CI: $0.97-1.99$ ). Incidences of extrapyramidal symptom-related adverse events (AEs) were $45.7 \%, 13.7 \%$, and $10.6 \%$ in the $\mathrm{COA}, \mathrm{AOA}$, and oral paliperidone/risperidone groups vs $23.9 \%$ in the PP group. Incidences of prolactin-related AEs were $5.7 \%, 3.8 \%$, and $3.5 \%$ vs $23.5 \%$, and incidences of $\geq 7 \%$ weight increase were $11.4 \%, 14.9 \%$, and $16.0 \%$ vs $32.4 \%$.

Conclusions. Results suggest a lower risk of TF but a higher rate of some AEs after treatment with PP vs COAs, AOAs, and paliperidone/risperidone. Deselection of specific OAs and low patient-compliance rates with OAs likely biased the safety results.

Received 21 January 2016; Accepted 29 March 2016; First published online 15 September 2016

Key words: Long-acting injectable antipsychotic, oral antipsychotic, paliperidone palmitate, PRIDE, schizophrenia.

\section{Introduction}

Schizophrenia is a chronic, severe, and often disabling mental illness characterized by deficits in thought processes, perceptions, and emotional responsiveness. ${ }^{1,2}$ The treatment of schizophrenia typically requires a multidisciplinary approach that involves both psychosocial

* Address for correspondence: Edward Kim, MD, MBA, Janssen Scientific Affairs, LLC, US Medical Affairs, 1125 Trenton-Harbourton Road, Titusville, NJ 08560, USA.

(Email: ekim37@its.jnj.com)

The authors thank Matthew Grzywacz, PhD, and Lynn Brown, PhD (ApotheCom, Yardley, PA), for their writing and editorial assistance. and psychopharmacological interventions. ${ }^{2}$ Over the past decade, advances and innovations in antipsychotic medications have enabled physicians to tailor treatment regimens according to patients' individual needs. For example, patients who have difficulty adhering to daily oral medication may benefit from using long-acting injectable (LAI) antipsychotic therapies, which provide therapeutic plasma concentrations over several weeks and ensure adherence. ${ }^{3-5}$ Although LAIs have been available for several years, clinical trials comparing the efficacy and safety of LAIs and oral antipsychotics have produced inconsistent results ${ }^{6-9}$ and therefore warrant further study. 
The atypical once-monthly LAI antipsychotic paliperidone palmitate (PP) is a nanocrystal formulation of paliperidone that dissolves slowly after intramuscular injection. ${ }^{10}$ The efficacy, safety, and tolerability of PP have been evaluated extensively in patients with schizophrenia in several short-term (ie, $\leq 6$ month) explanatory trials. ${ }^{3,4,11-22}$ Fewer studies have examined the longer-term (ie, $>6$ month) efficacy and safety of $\mathrm{PP},{ }^{18,23-28}$ and because these trials were primarily explanatory in design, their results do not necessarily reflect real-world situations. ${ }^{27}$ Additional long-term studies that evaluate real-world outcomes are needed to better understand both the efficacy and effectiveness of PP in patients with schizophrenia.

The Paliperidone Palmitate Research in Demonstrating Effectiveness (PRIDE) study ${ }^{27,29}$ is a 15-month, prospective, randomized study comparing the effects of PP with those of daily oral antipsychotics (ie, haloperidol, perphenazine, olanzapine, aripiprazole, quetiapine, risperidone, and paliperidone) on treatment failure in a trial designed to reflect real-world schizophrenia patients, treatments, and outcomes. PRIDE is unique in that it incorporates both explanatory (efficacy) and pragmatic (effectiveness) design elements. PRIDE included patients traditionally excluded from randomized trials, such as those with a history of incarceration and comorbid substance abuse; allowed flexibility in treatment and management decisions; and included a range of real-world consequences as endpoints (ie, arrest/incarceration, psychiatric hospitalization, suicide, discontinuation due to inadequate efficacy or intolerability, treatment supplementation due to inadequate efficacy, or increased psychiatric services to prevent hospitalization). The primary results of PRIDE showed that treatment with once-monthly PP significantly delayed treatment failure compared with daily oral antipsychotics in the overall study population (hazard ratio [HR]: 1.43 ; 95\% confidence interval [CI]: 1.09-1.88; $\log$-rank $\mathrm{p}=0.011$ ); the median event time was 190 days longer in the PP group, and there was a $43 \%$ reduction in risk of treatment failure relative to oral antipsychotics. ${ }^{29}$

The PRIDE study's randomization scheme provides a sampling basis for exploratory comparisons of once-monthly PP to classes of oral antipsychotic medications. We hypothesized that the significant delay in treatment failure observed with once-monthly PP would be observed when compared with subgroups of conventional oral antipsychotics (ie, haloperidol and perphenazine) and atypical oral antipsychotics (ie, aripiprazole, olanzapine, quetiapine, paliperidone, and risperidone). Additionally, we sought to compare the efficacy and safety of once-monthly PP with oral paliperidone and oral risperidone to examine outcomes following treatment with different delivery methods of the same molecule platform (ie, LAI versus oral delivery of risperidone and its active metabolite, paliperidone).

\section{Methods}

\section{Study design}

The detailed methodology has been published elsewhere. $^{27,29}$ In brief, PRIDE (NCT01157351) was a randomized, prospective, open-label, event-monitoring board-blinded, parallel-group study that compared once-monthly PP and oral antipsychotics on treatment failure in subjects with schizophrenia and a history of incarceration. The study included a screening phase of up to 2 weeks, followed by a 15-month randomized, open-label treatment phase. ${ }^{27}$ The study was conducted between May 5, 2010, and December 9, 2013, at 50 sites across 25 U.S. states and Puerto Rico. ${ }^{29}$ The study protocol was approved by each site's institutional review board and was conducted in accordance with the ethical principles established by the Declaration of Helsinki.

\section{Participants}

Adults aged 18 to 65 years with a current diagnosis of schizophrenia (Diagnostic and Statistical Manual of Mental Disorders, Fourth Edition criteria ${ }^{30}$ that was confirmed by the M.I.N.I. International Neuropsychiatric Interview, version $6.0^{31}$ ) were eligible for study participation. Subjects must have been taken into custody by the criminal justice system $\geq 2$ times in the previous 2 years, with $\geq 1$ of these events leading to incarceration; have been released from most recent custody within 90 days of the screening visit; and be willing to use a once-monthly LAI antipsychotic. ${ }^{29}$ All subjects provided written informed consent.

\section{Interventions}

Study interventions included paliperidone palmitate and oral antipsychotic medications. ${ }^{29}$ Seven oral antipsychotic medications were available to participants: haloperidol, perphenazine, olanzapine, aripiprazole, quetiapine, risperidone, and paliperidone. Before random treatment assignment, subjects reviewed these oral medication options with their physicians to determine acceptability based on prior experience. Up to 6 oral antipsychotic medications could be deselected by the subject or the physician, and reasons for deselection were documented.

\section{Randomization}

An equipoise stratified randomization scheme ${ }^{32}$ was used for treatment assignment. ${ }^{29}$ The equipoise strata were defined by the sets of acceptable oral antipsychotic medications selected by subjects and their physicians before randomization. Subjects were randomized within their equipoise stratum in a 1:1 ratio to treatment with flexibly dosed once-monthly PP (78-234 mg) or a flexibly dosed oral antipsychotic that was randomly selected from the oral 
medications in the equipoise stratum. For example, subjects who selected all 5 atypical antipsychotics as acceptable treatment options were randomized according to a randomization schedule allocated for the atypical antipsychotic stratum, subjects who selected the 2 conventional antipsychotics were randomized according to a randomization schedule allocated for the conventional antipsychotic stratum, and so on. The randomization scheme provided a sampling basis for comparing multiple treatment options. For example, to compare PP versus conventional antipsychotics, subjects who were randomized would be pooled from the strata that included 1 or more conventional antipsychotics.

\section{Study medications}

Flexible monthly maintenance doses of once-monthly PP were given according to the product label in a dose range of $78-234 \mathrm{mg}$ (50-150 $\mathrm{mg}$ equivalents); the recommended target maintenance dose was $156 \mathrm{mg} .{ }^{29}$ Oral antipsychotic doses were selected and adjusted within the dose range of the package insert; occasional dosing outside the range specified in the package insert was allowed. The use of nonantipsychotic psychotropic medications (ie, mood stabilizers, antidepressants, anxiolytics, or hypnotics) was permitted if clinically indicated as concomitant therapy; however, monotherapy with the assigned study drug was encouraged. Subjects who discontinued study treatment or experienced treatment failure and did not withdraw consent could continue to be followed through month 15 of the treatment phase.

\section{Study end points}

For this analysis we used the primary study endpoint, time to treatment failure, which was defined as any of the following events: arrest or incarceration, psychiatric hospitalization, suicide, discontinuation of treatment due to inadequate efficacy (in the investigator's opinion), treatment supplementation with another antipsychotic due to inadequate efficacy, discontinuation of treatment due to safety or tolerability, or increase in psychiatric services to prevent imminent psychiatric hospitalization. ${ }^{29}$ First treatment failure events were initially assessed by site investigators, and each event was subsequently reviewed and adjudicated as determined by an independent event-monitoring board that was blinded to individual subject treatment assignment. Exploratory analyses of time to treatment failure compared the following: (1) once-monthly PP versus daily conventional oral antipsychotics (haloperidol or perphenazine), (2) oncemonthly PP versus daily atypical oral antipsychotics (olanzapine or aripiprazole or quetiapine or paliperidone or risperidone), and (3) once-monthly PP versus daily oral paliperidone plus risperidone.

\section{Statistical analyses}

Statistical analyses were the same as those used for the primary analysis. ${ }^{29}$ The intent-to-treat (ITT) population, defined as all randomly assigned subjects who received $\geq 1$ dose of their study treatment, was used for efficacy and safety analyses. Event-free probabilities were estimated using the Kaplan-Meier method; treatment differences were assessed using a log-rank test based on ITT analysis set; HRs and 95\% CIs were estimated using Cox proportional hazards regression models, with randomly assigned treatment as a fixed factor. The number needed to treat (NNT) was calculated as the inverse of absolute risk reduction, defined as the difference in estimated event rates at month 15 between treatment groups. The differences across subgroups regarding adverse event (AE) outcomes were reported descriptively. The differences in demographics and baseline variables between treatment subgroups were assessed using the Wilcoxon rank-sum test for continuous variables or Fisher's exact test for categorical variables. No adjustment was made for multiplicity.

\section{Results}

\section{Reasons for deselection of oral antipsychotic medications}

Reasons for the deselection of oral antipsychotic medications prior to the start of the study are shown in Table 1. Haloperidol (59.9\%) and perphenazine (39.4\%) were the most commonly deselected oral antipsychotics prior to randomization, primarily because of extrapyramidal symptom (EPS)-related AEs. Paliperidone was deselected less than any other oral antipsychotic (7.7\%). For atypical oral antipsychotics, the most common deselection reasons were weight gain and other.

\section{Patient disposition and baseline characteristics}

In the primary analysis, 450 subjects were randomly assigned to PP ( $\mathrm{n}=230,51.1 \%)$ or oral antipsychotics ( $\mathrm{n}=220,48.9 \%$ ) and 444 subjects were included in the ITT population. ${ }^{29}$ Of these, 226 (50.9\%) received PP and $218(49.1 \%)$ received oral antipsychotics (183 [83.9\%], atypical oral antipsychotics; 35 [16.1\%], conventional oral antipsychotics) (Figure 1). Sample sizes for the PP versus conventional oral antipsychotic comparison, the PP versus atypical oral antipsychotic comparison, and the PP vs paliperidone plus risperidone comparison are also shown in Figure 1. Baseline demographics and clinical characteristics were generally similar (ITT population) (Table 2). The mean (SD) daily doses (mg) of prescribed oral antipsychotics were $8.2(5.33)$, 16.5 (8.81), 13.3 (6.44), 15.3 (5.89), 339.9 (180.35), $3.6(1.61)$, and 6.6 (2.44) for haloperidol, perphenazine, olanzapine, aripiprazole, quetiapine, risperidone, and 
TABLE 1. Reasons for deselection of oral antipsychotic medications at study start in total study population ( $\mathrm{n}=444$ ) (intent-to-treat population)

\begin{tabular}{|c|c|c|c|c|c|c|c|}
\hline & \multicolumn{2}{|c|}{ Conventional antipsychotics } & \multicolumn{5}{|c|}{ Atypical antipsychotics } \\
\hline & Haloperidol & Perphenazine & Olanzapine & Aripiprazole & Quetiapine & Paliperidone & Risperidone \\
\hline Subjects not selecting the specific oral antipsychotic agent, $n(\%)$ & $266(59.9)$ & $175(39.4)$ & $147(33.1)$ & $119(26.8)$ & $144(32.4)$ & $34(7.7)$ & $90(20.3)$ \\
\hline \multicolumn{8}{|l|}{ Reasons for deselection } \\
\hline EPS (Parkinsonism, dystonia, akathisia) & $160(36.0)$ & $91(20.5)$ & $5(1.1)$ & $12(2.7)$ & $6(1.4)$ & $1(0.2)$ & $18(4.1)$ \\
\hline EPS (Tardive dyskinesia) & $71(16.0)$ & $54(12.2)$ & $5(1.1)$ & $3(0.7)$ & $1(0.2)$ & $2(0.5)$ & $6(1.4)$ \\
\hline Inadequate efficacy & $10(2.3)$ & $4(0.9)$ & $12(2.7)$ & $32(7.2)$ & $10(2.3)$ & $2(0.5)$ & $18(4.1)$ \\
\hline Prolactin elevation/sexual AEs & $14(3.2)$ & $8(1.8)$ & $8(1.8)$ & $5(1.1)$ & $6(1.4)$ & $3(0.7)$ & $12(2.7)$ \\
\hline Anticholinergic AEs & $10(2.3)$ & $5(1.1)$ & $2(0.5)$ & $7(1.6)$ & $19(4.3)$ & $1(0.2)$ & $9(2.0)$ \\
\hline Cardiovascular AEs & $3(0.7)$ & $3(0.7)$ & $22(5.0)$ & $14(3.2)$ & $10(2.3)$ & $1(0.2)$ & $9(2.0)$ \\
\hline Weight gain & $1(0.2)$ & $3(0.7)$ & $83(18.7)$ & $22(5.0)$ & $34(7.7)$ & $3(0.7)$ & $9(2.0)$ \\
\hline Other & $48(10.8)$ & $44(9.9)$ & $33(7.4)$ & $38(8.6)$ & $75(16.9)$ & $23(5.2)$ & $22(5.0)$ \\
\hline
\end{tabular}

Abbreviations: AEs, adverse events; EPS, extrapyramidal symptoms.

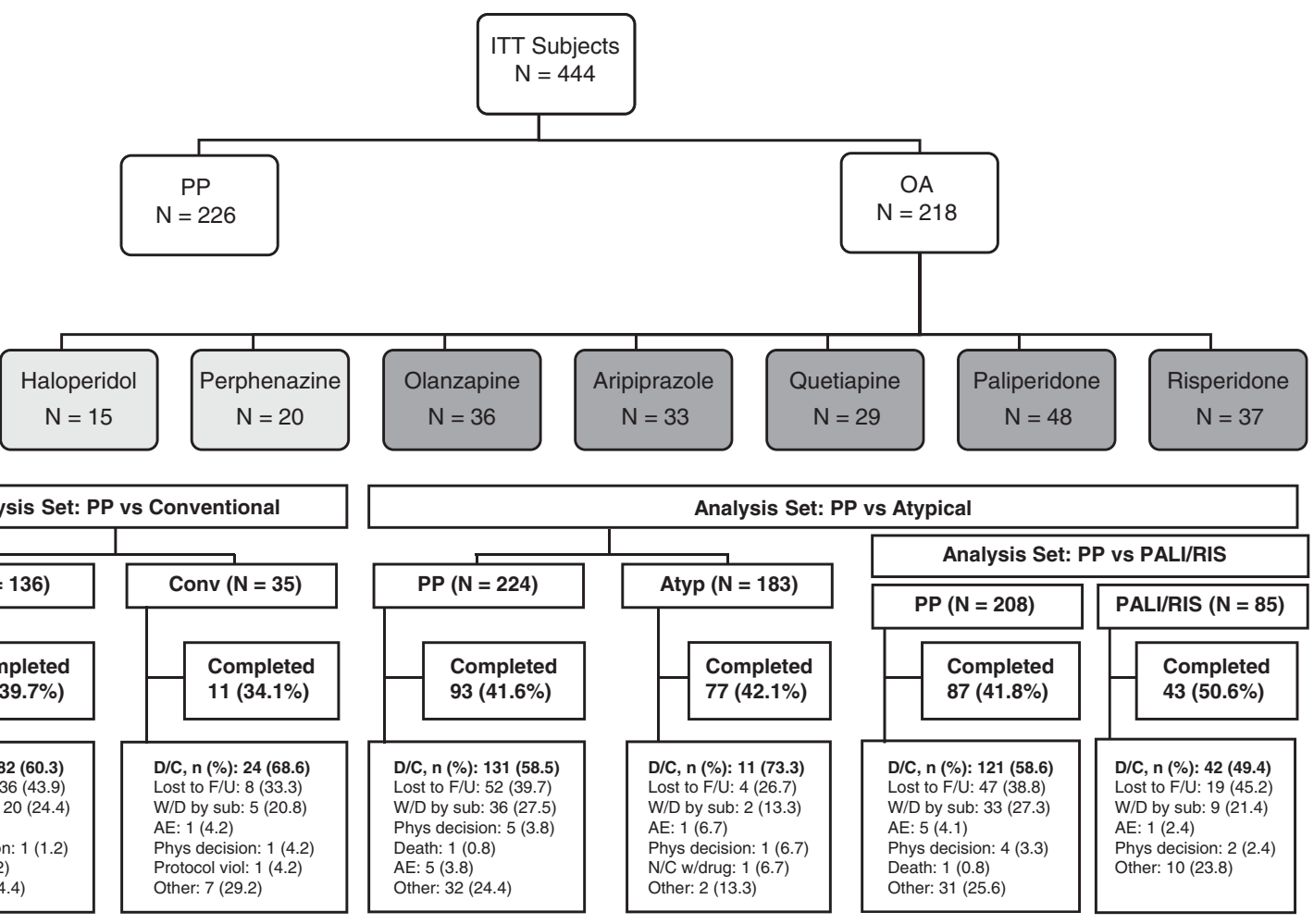

FIGURE 1. Study flow diagram and analysis sets. Abbreviations: AE, adverse event; AP, antipsychotic; Atyp, atypical antipsychotic; Conventional/Conv, conventional antipsychotic; D/C, discontinued; F/U, follow-up; N/C, noncompliance; OA, oral antipsychotic; PALI/RIS, paliperidone/risperidone; Phys, physician; $\mathrm{PP}$, paliperidone palmitate; sub, subject; viol, violation; W/D, withdrawal.

paliperidone, respectively. The mean (SD) monthly PP dose was $181.3(34.19) \mathrm{mg}$.

\section{First treatment failure}

Once-monthly PP versus conventional oral antipsychotics

In total, 136 subjects receiving once-monthly PP and 35 subjects receiving conventional oral antipsychotics were included in this analysis. Treatment failures occurred in $62(45.6 \%)$ subjects in the PP group and in 19 (54.3\%) subjects in the conventional oral antipsychotic group. The estimated event rate at month 15 was $57.0 \%$ in the PP group and $66.0 \%$ in the conventional oral group $(\mathrm{NNT}=11)$. The risk for treatment failure was $34 \%$ higher with conventional oral antipsychotics (HR: 1.34; 95\% CI: $0.80-2.25 ; \mathrm{p}=0.262$ ) than with once-monthly 


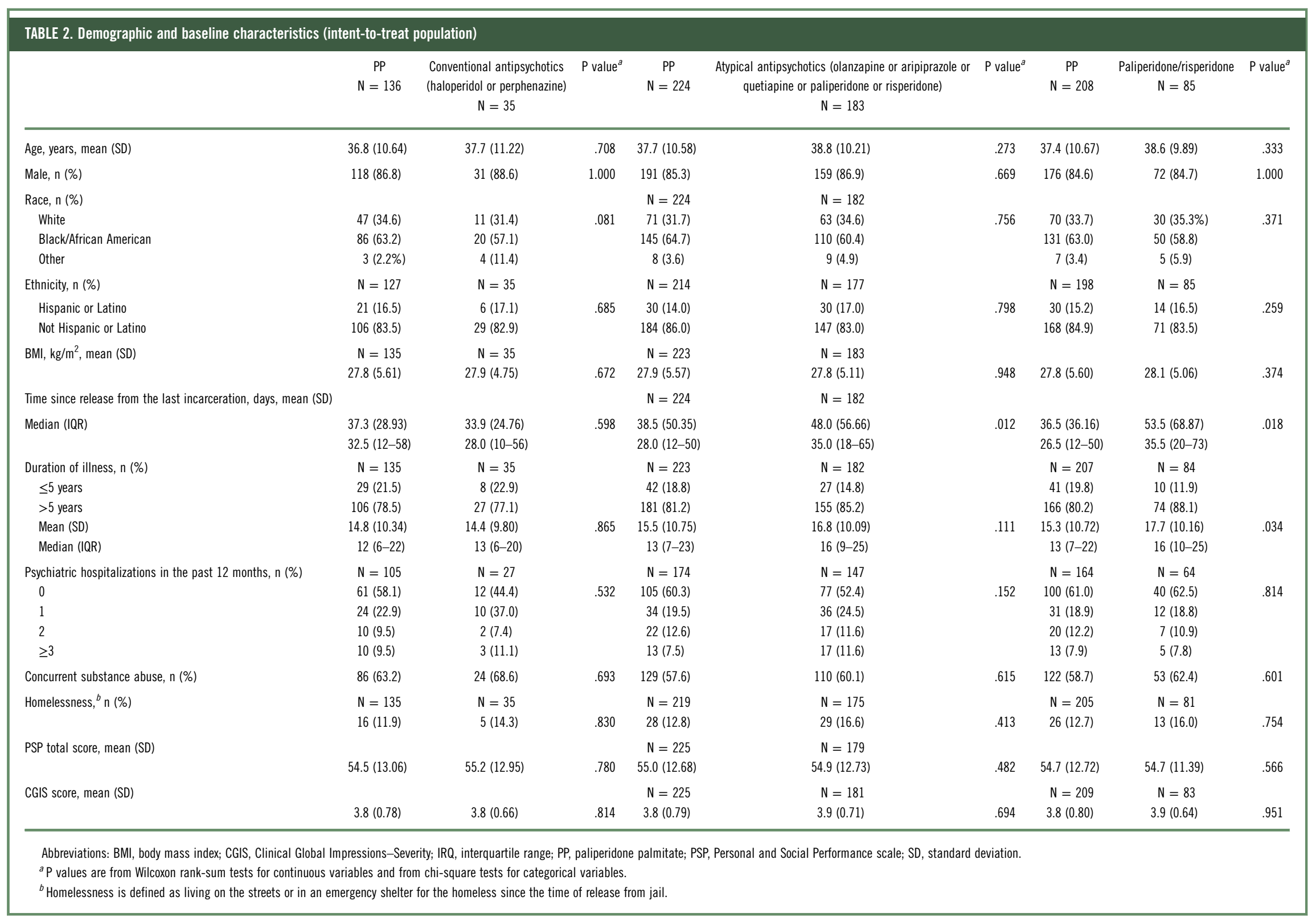


PP (Figure 2A). The median number of days to treatment failure was 302 days for PP and 142 days for conventional oral antipsychotics.

\section{Once-monthly PP versus atypical oral antipsychotics}

In total, 224 subjects receiving once-monthly PP and 183 subjects receiving atypical oral antipsychotics were included in this analysis. Eighty-nine subjects (39.7\%) in the PP group and 98 (53.6\%) subjects in the atypical oral antipsychotic group had a treatment failure event. The estimated event rate at month 15 was $52 \%$ in the PP group and $63 \%$ in the atypical oral antipsychotic group $(\mathrm{NNT}=9)$. The risk for treatment failure was $41 \%$ higher with atypical oral antipsychotics (HR: 1.41; 95\% CI: $1.06-1.88 ; \mathrm{p}=0.019$ ) than with PP (Figure 2B). The median number of days to treatment failure was 428 days for PP and 229 days for atypical oral antipsychotics.

\section{Once-monthly PP versus paliperidone/risperidone}

A total of 208 subjects receiving once-monthly PP and 85 subjects receiving oral paliperidone/risperidone were included in this analysis. Treatment failures occurred in 85 (40.9\%) subjects in the PP group and in 46 (54.1\%) subjects in the paliperidone/risperidone group. The estimated event rate at month 15 was $53 \%$ in the PP group and $64 \%$ in the oral paliperidone/risperidone group $(\mathrm{NNT}=9)$. The risk for treatment failure was $39 \%$ higher in the paliperidone/risperidone group (HR: 1.39; 95\% CI: 0.97-1.99; $\mathrm{p}=0.071$ ) than with PP (Figure 2C). The median number of days to treatment failure was 416 days for PP and 229 days for paliperidone/ risperidone.

\section{Once-monthly PP versus individual oral antipsychotics}

Comparisons of once-monthly PP to individual oral antipsychotics lacked the power to detect statistically significant differences. However, there was a trend toward better efficacy with once-monthly PP, including versus oral delivery of paliperidone (Supplementary Figure 1, available online).

\section{Reasons for first treatment failure}

In all 3 subgroup analyses, arrest/incarceration (range, $20.0 \%-31.1 \%$ ) and psychiatric hospitalization (range, 8.0\%-15.3\%) were the most common reasons for first treatment failure (Table 3 ). No suicides were reported.

\section{Safety}

Treatment-emergent AEs (TEAEs) were reported in $91.4 \%, 77.6 \%$, and $77.6 \%$ of subjects in the conventional antipsychotic, atypical antipsychotic, and paliperidone plus risperidone groups, respectively (Table 4). The frequency of TEAEs in the once-monthly PP groups ranged from $85.7 \%$ to $87.0 \%$.

Serious AEs occurred in $20.0 \%, 21.9 \%$, and $24.7 \%$ of subjects in the conventional antipsychotic, atypical antipsychotic, and paliperidone plus risperidone groups, respectively. The incidence of serious AEs ranged from $17.4 \%$ to $19.9 \%$ in the once-monthly PP groups. TEAEs leading to study drug discontinuation occurred in $22.9 \%$, $4.9 \%$, and $7.1 \%$ of subjects in the conventional antipsychotic, atypical antipsychotic, and paliperidone/ risperidone groups, respectively, and ranged from $11.5 \%$ to $12.1 \%$ in the once-monthly PP groups.

EPS-related TEAEs were reported more frequently by subjects in the conventional antipsychotic group $(45.7 \%)$ compared with those in the atypical antipsychotic $(13.7 \%)$, paliperidone plus risperidone $(10.6 \%)$, or once-monthly PP (range, 24.1\%-25.0\%) groups (Table 4). Prolactin-related TEAEs were more commonly reported in the once-monthly PP groups (range, 23.7\%$25.0 \%$ ) than in the oral antipsychotic groups (range, $3.5 \%-5.7 \%$ ). Alphs et $a l^{29}$ reported on an increased incidence of prolactin-related TEAEs with once-monthly PP vs oral antipsychotics that was apparent in both males and females. $\mathrm{A} \geq 7 \%$ increase in body weight at month 15 (last observation carried forward) was observed in approximately one-third of subjects who received oncemonthly PP (range, 32.3\%-33.8\%) compared with $11.4 \%, 14.9 \%$, and $16.0 \%$ of subjects in the conventional, atypical, and paliperidone/risperidone groups. One death occurred in the PP group and was considered by the investigator as unlikely related to study drug.

\section{Discussion}

These exploratory analyses from the PRIDE study show that the risk of treatment failure was $34 \%$ and $41 \%$ higher with oral conventional antipsychotics and oral atypical antipsychotics, respectively, when compared with PP. Although subgroup comparison with oral conventional antipsychotics did not reach statistical significance, which was likely due to small and underpowered subsamples, the effectiveness results were consistent with the primary analysis of PRIDE $^{29}$ and support the premise that improvements observed with PP are consistent across classes of treatments (oral conventional antipsychotics and oral atypical antipsychotics). Our exploratory analyses also evaluated the effect of delivery method on outcome. Compared with PP, the risk for treatment failure was $39 \%$ higher with oral delivery of similar or identical molecules (paliperidone/risperidone), suggesting that there may be differences in the effectiveness between the 2 formulations. The primary driver of this difference may be attributable to the longer half-life of $\mathrm{PP}$, which provides 


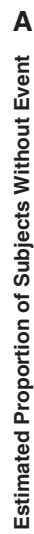

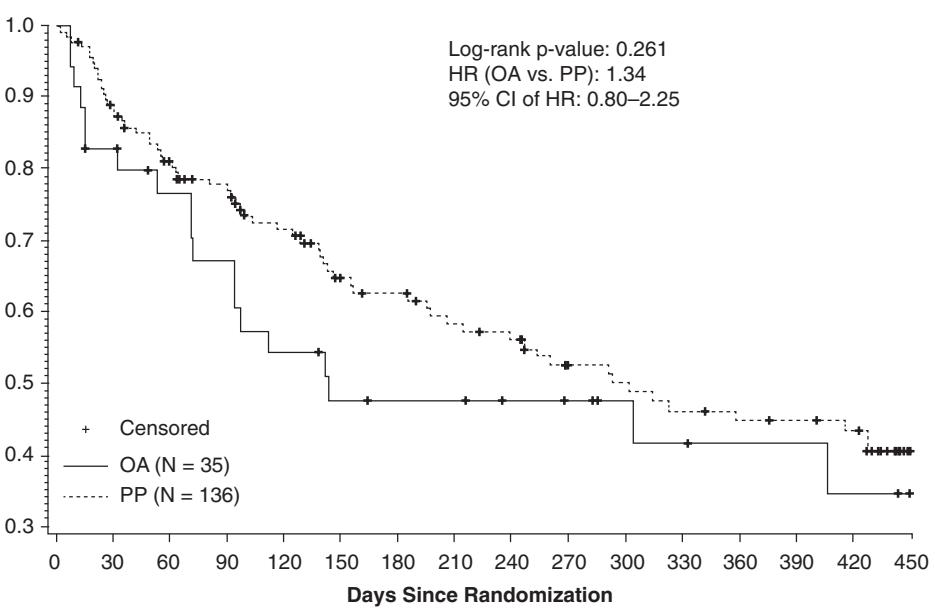

Number of subjects at risk

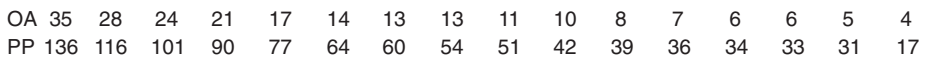

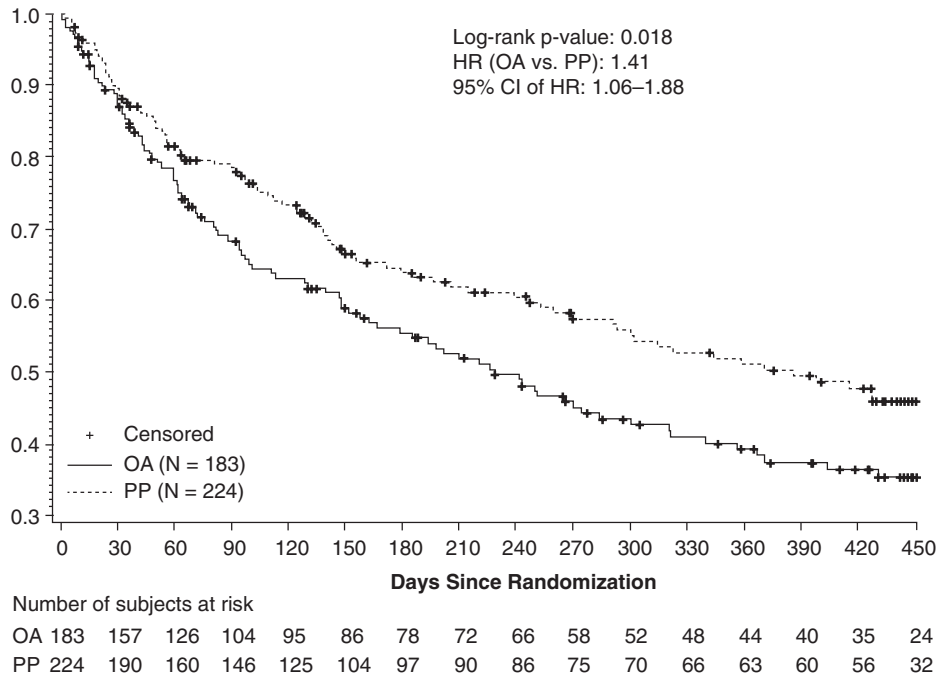

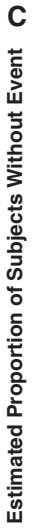

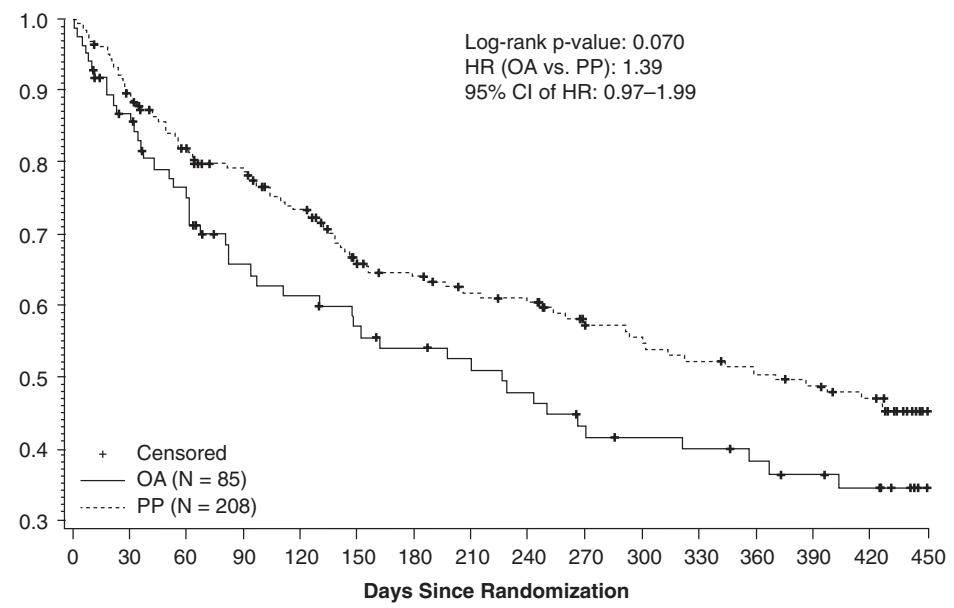

Number of subjects at risk

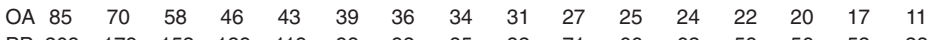

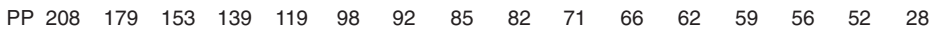

FIGURE 2. Kaplan-Meier estimates of time to first treatment failure. (A) PP vs conventional antipsychotics (haloperidol or perphenazine). (B) PP vs atypical OAs (paliperidone, risperidone, olanzapine, aripiprazole, or quetiapine). (C) PP vs paliperidone/risperidone. Abbreviations: $\mathrm{Cl}$, confidence interval; HR, hazard ratio; $\mathrm{OA}$, oral antipsychotic; PP, paliperidone palmitate. 
TABLE 3. Reasons for first treatment failure (intent-to-treat population)

\begin{tabular}{|c|c|c|c|c|c|c|}
\hline & $\begin{array}{c}\text { PP } \\
n=136\end{array}$ & $\begin{array}{c}\text { Conventional } \\
\text { antipsychotics } \\
\text { (haloperidol or } \\
\text { perphenazine) } \\
\quad \mathrm{n}=35\end{array}$ & $\begin{array}{c}P P \\
n=224\end{array}$ & $\begin{array}{l}\text { Atypical antipsychotics (olanzapine } \\
\text { or aripiprazole or quetiapine or } \\
\text { paliperidone or risperidone) } \\
\qquad n=183\end{array}$ & $\begin{array}{c}P P \\
n=208\end{array}$ & $\begin{array}{l}\text { Atypical antipsychotics } \\
\text { (paliperidone/risperidone) } \\
\qquad n=85\end{array}$ \\
\hline Any event, $\mathrm{n}(\%)$ & $62(45.6)$ & $19(54.3)$ & $89(39.7)$ & $98(53.6)$ & $85(40.9)$ & $46(54.1)$ \\
\hline \multicolumn{7}{|l|}{ Reason for first treatment failure, $n(\%)$} \\
\hline Arrest/incarceration & $35(25.7)$ & $7(20.0)$ & $47(21.0)$ & 57 (31.1) & $45(21.6)$ & $24(28.2)$ \\
\hline Psychiatric hospitalization & $11(8.1)$ & $3(8.6)$ & $18(8.0)$ & $23(12.6)$ & $17(8.2)$ & $13(15.3)$ \\
\hline $\begin{array}{l}\text { Discontinuation of antipsychotic treatment due to } \\
\text { safety or tolerability }\end{array}$ & $9(6.6)$ & $3(8.6)$ & $15(6.7)$ & $5(2.7)$ & $14(6.7)$ & $4(4.7)$ \\
\hline $\begin{array}{l}\text { Treatment supplementation with another } \\
\text { antipsychotic due to inadequate efficacy }\end{array}$ & $4(2.9)$ & $2(5.7)$ & $5(2.2)$ & $4(2.2)$ & $5(2.4)$ & $2(2.4)$ \\
\hline $\begin{array}{l}\text { Discontinuation of antipsychotic treatment due to } \\
\text { inadequate efficacy }\end{array}$ & $1(0.7)$ & $4(11.4)$ & $1(0.4)$ & $5(2.7)$ & $1(0.5)$ & $1(1.2)$ \\
\hline $\begin{array}{l}\text { Increase in level of psychiatric services to prevent } \\
\text { imminent psychiatric hospitalization }\end{array}$ & $2(1.5)$ & 0 & $3(1.3)$ & $4(2.2)$ & $3(1.4)$ & $2(2.4)$ \\
\hline Suicide & 0 & 0 & 0 & 0 & 0 & 0 \\
\hline
\end{tabular}

a longer duration of continuous effective exposure than the oral formulation. ${ }^{33}$ Unlike oral medications, LAI formulations provide the physician with certain knowledge of adherence and with greater opportunity to correct nonadherence before marked declines in plasma levels occur. ${ }^{34}$

The overall higher incidence of AEs observed in PRIDE was likely due to its somewhat unique study design. In studies where patients are stabilized before randomization, patients may drop out due to AEs before randomization occurs. Patients in PRIDE were followed from the first dose; therefore, the duration of $\mathrm{AE}$ reporting was longer compared with that of standard trial designs. As expected, the greater adherence and medication exposure associated with an LAI contribute to differing $\mathrm{AE}$ profiles compared to those for oral antipsychotics. Differential AE profiles include higher rates of injection site pain, prolactin-related AEs, and weight gain associated with PP, and higher rates of EPS-related AEs associated with oral conventional antipsychotics. ${ }^{35-37}$ These findings support the knowledge that conventional oral antipsychotics have clinically relevant EPS-related AEs that may limit their clinical utility. It must be considered, however, that $\mathrm{AE}$ reporting rates may reflect true differences, greater adherence with $\mathrm{PP}$, and/or biases introduced by the deselection process for this study, which would favor patients who deselected oral antipsychotics that had led to relevant AEs in the past. For these reasons, the unique study design of PRIDE likely impacted AE profiles. For example, in the PROSIPAL study, which evaluated 24-month outcomes of patients treated with PP compared with oral antipsychotics (aripiprazole, quetiapine, olanzapine, paliperidone extended release, risperidone, or haloperidol) in patients with recently diagnosed schizophrenia, rates of prolactin AEs were similar at $6.3 \%$ and $5.0 \%$, respectively. ${ }^{38}$ Similarly, a post hoc analysis that compared data from a PP trial (median of 170 days of exposure) and an oral paliperidone extended-release trial (median of 45 days of exposure) showed comparable rates of TEAEs of interest, including prolactin-related AEs $(2.1 \%$ and $2.9 \%$, respectively). ${ }^{39}$ Owing to differences in trial design, caution must be used when comparing PRIDE to other studies.

Several limitations should be considered when interpreting these data. First, although findings from PRIDE are more reflective of real-world outcomes than trials with extensive exclusion and inclusion criteria, these data cannot be generalized to all patients with schizophrenia. Indeed, subpopulations may have more or less effect. Second, subgroup analyses had lower $\mathrm{N}$ values than the overall analysis, and caution should be exercised when interpreting findings in subgroups. Third, despite randomization, the preselection of suitable oral antipsychotics before randomization could have introduced selection bias in favor of the oral antipsychotic group because only the oral medication(s) selected could be randomly assigned if patients were not randomly assigned to the PP arm. The deselection of specific oral antipsychotics as well as low compliance with oral antipsychotics may have biased the safety results, masking tolerability issues associated with any individual oral antipsychotic. Fourth, due to the nature of exploratory analyses, multiplicity adjustments were not performed; hence, the overall type I error would be greater than $5 \%$, the nominal level used for each individual test.

Collectively, these data suggest that PP confers effectiveness advantages over oral antipsychotic therapies. 


\begin{tabular}{|c|c|c|c|c|c|c|}
\hline & \multicolumn{2}{|c|}{ PP vs conventional antipsychotics } & \multicolumn{2}{|r|}{ PP vs atypical antipsychotics } & \multicolumn{2}{|c|}{ PP vs paliperidone + risperidone } \\
\hline & $\begin{array}{c}\text { PP } \\
n=136\end{array}$ & $\begin{array}{l}\text { Conventional antipsychotics } \\
\text { (haloperidol or perphenazine) } \\
\qquad n=35\end{array}$ & $\begin{array}{c}P P \\
n=224\end{array}$ & $\begin{array}{l}\text { Atypical antipsychotics (olanzapine or aripiprazole or } \\
\text { quetiapine or paliperidone or risperidone) } \\
\qquad \mathrm{n}=183\end{array}$ & $\begin{array}{c}\text { PP } \\
\mathrm{n}=208\end{array}$ & $\begin{array}{l}\text { Atypical antipsychotics } \\
\text { (paliperidone or risperidone) } \\
\qquad \mathrm{n}=85\end{array}$ \\
\hline \multicolumn{7}{|l|}{ TEAE, n (\%) } \\
\hline Any & $118(86.8)$ & $32(91.4)$ & $192(85.7)^{*}$ & $142(77.6)$ & $181(87.0)$ & $66(77.6)$ \\
\hline Injection site pain & $23(16.9)^{*}$ & 0 & $42(18.8)^{*}$ & 0 & $40(19.2)^{*}$ & 0 \\
\hline Insomnia & $25(18.4)$ & $2(5.7)$ & $38(17.0)$ & $23(12.6)$ & $37(17.8)$ & $9(10.6)$ \\
\hline Weight increased & $10(7.4)$ & 0 & $26(11.6)$ & $13(7.1)$ & $23(11.1)$ & $9(10.6)$ \\
\hline Akathisia & $17(12.5)$ & $6(17.1)$ & $25(11.2)^{*}$ & $9(4.9)$ & $24(11.5)^{*}$ & $2(2.4)$ \\
\hline Anxiety & $14(10.3)$ & $3(8.6)$ & $23(10.3)$ & $13(7.1)$ & $23(11.1)$ & $8(9.4)$ \\
\hline Depression & $11(8.1)$ & $1(2.9)$ & $17(7.6)$ & $13(7.1)$ & $16(7.7)$ & $6(7.1)$ \\
\hline Fatigue & $9(6.6)$ & 0 & $17(7.6)$ & $6(3.3)$ & $14(6.7)$ & $3(3.5)$ \\
\hline Erectile dysfunction & $11(8.1)^{\mathrm{b}}$ & 0 & $17(7.6)^{\star}$ & 0 & $16(7.7)^{\star}$ & 0 \\
\hline Sedation & $11(8.1)$ & $2(5.7)$ & $15(6.7)$ & $14(7.7)$ & $15(7.2)$ & $5(5.9)$ \\
\hline Dry mouth & $11(8.1)$ & $3(8.6)$ & $15(6.7)$ & $15(8.2)$ & $14(6.7)$ & $7(8.2)$ \\
\hline Increased appetite & $9(6.6)$ & $1(2.9)$ & $15(6.7)$ & $7(3.8)$ & $14(6.7)$ & $4(4.7)$ \\
\hline Nasopharyngitis & $9(6.6)$ & $4(11.4)$ & $15(6.7)$ & $8(4.4)$ & $15(7.2)$ & $4(4.7)$ \\
\hline Headache & $9(6.6)$ & $3(8.6)$ & $14(6.3)$ & $15(8.2)$ & $11(5.3)$ & $8(9.4)$ \\
\hline Libido decreased & $8(5.9)$ & $1(2.9)$ & $13(5.8)^{\star}$ & $2(1.1)$ & $13(6.3)$ & $1(1.2)$ \\
\hline Upper respiratory tract infection & $5(3.7)$ & 0 & $13(5.8)$ & $10(5.5)$ & $13(6.3)$ & $7(8.2)$ \\
\hline Back pain & $9(6.6)$ & $1(2.9)$ & $13(5.8)$ & $7(3.8)$ & $12(5.8)$ & $5(5.9)$ \\
\hline Schizophrenia & $8(5.9)$ & $4(11.4)$ & $10(4.5)$ & $11(6.0)$ & $10(4.8)$ & $5(5.9)$ \\
\hline Somnolence & $6(4.4)$ & $4(11.4)$ & $10(4.5)$ & $11(6.0)$ & $8(3.8)$ & $2(2.4)$ \\
\hline Toothache & $7(5.1)$ & $2(5.7)$ & $10(4.5)$ & $10(5.5)$ & $8(3.8)$ & $3(3.5)$ \\
\hline Dizziness & $1(0.7)$ & $4(11.4)^{*}$ & $5(2.2)$ & $7(3.8)$ & $4(1.9)$ & $4(4.7)$ \\
\hline Suicidal ideation & $6(4.4)$ & $2(5.7)$ & $8(3.6)$ & $11(6.0)$ & $7(3.4)$ & $4(4.7)$ \\
\hline Agitation & $5(3.7)$ & $2(5.7)$ & $6(2.7)$ & $3(1.6)$ & $6(2.9)$ & $3(3.5)$ \\
\hline Hallucination, auditory & $2(1.5)$ & $2(5.7)$ & $4(1.8)$ & $5(2.7)$ & $4(1.9)$ & $3(3.5)$ \\
\hline Dyskinesia & $4(2.9)$ & $2(5.7)$ & $6(2.7)$ & $1(0.5)$ & $6(2.9)$ & $1(1.2)$ \\
\hline Dystonia & $3(2.2)$ & $3(8.6)$ & $5(2.2)$ & $3(1.6)$ & $5(2.4)$ & $1(1.2)$ \\
\hline Extrapyramidal disorder & $3(2.2)$ & $2(5.7)$ & $4(1.8)$ & $2(1.1)$ & $3(1.4)$ & $1(1.2)$ \\
\hline Parkinsonism & $3(2.2)$ & $2(5.7)$ & $4(1.8)$ & $2(1.1)$ & $3(1.4)$ & $2(2.4)$ \\
\hline Tardive dyskinesia & $1(0.7)$ & $2(5.7)$ & $1(0.4)$ & $1(0.5)$ & $1(0.5)$ & 0 \\
\hline Vomiting & $2(1.5)$ & $2(5.7)$ & $3(1.3)$ & $3(1.6)$ & $3(1.4)$ & $2(2.4)$ \\
\hline Laceration & $3(2.2)$ & $3(8.6)$ & $4(1.8)$ & $5(2.7)$ & $4(1.9)$ & $4(4.7)$ \\
\hline Arthropod bite & $1(0.7)$ & $2(5.7)$ & $2(0.9)$ & $2(1.1)$ & $2(1.0)$ & 0 \\
\hline Abnormal weight gain & $9(6.6)$ & 0 & $11(4.9)$ & $3(1.6)$ & $11(5.3)$ & $2(2.4)$ \\
\hline Hypertension & 0 & $2(5.7)$ & $2(0.9)$ & $5(2.7)$ & $1(0.5)$ & $5(5.9)$ \\
\hline Psychotic disorder & $5(3.7)$ & $1(2.9)$ & $10(4.5)$ & $7(3.8)$ & $9(4.3)$ & $5(5.9)$ \\
\hline Irritability & $1(0.7)$ & 0 & $3(1.3)$ & $5(2.7)$ & $3(1.4)$ & $5(5.9)$ \\
\hline
\end{tabular}




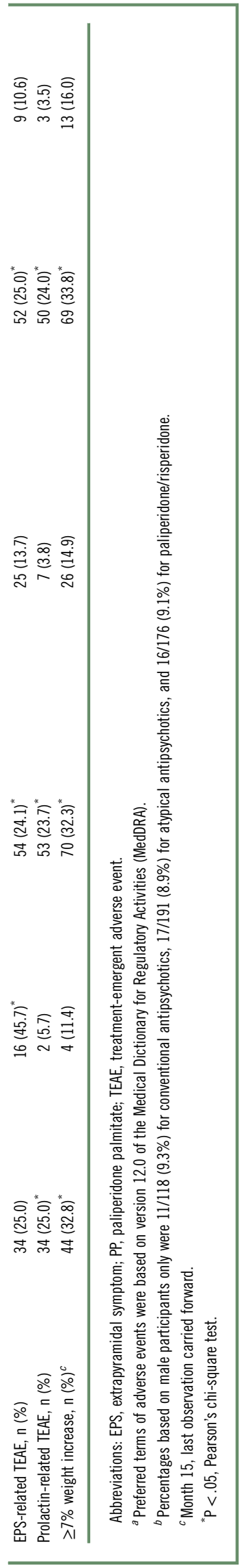

However, in the current study design, these advantages seemed to be associated with greater patient-reported AEs (except for lower EPS-related AEs vs the conventional oral antipsychotic group). Because PRIDE included patients normally excluded from clinical trials (eg, those with a history of contact with the criminal justice system), these findings are particularly noteworthy, as they reflect patients with real-world characteristics who were treated in realworld paradigms using real-world outcomes. Given the unique study design of PRIDE, additional analytic approaches may be explored, such as covariate analyses that adjust for baseline characteristics and time-dependent variables.

\section{Conclusions}

Exploratory analyses of PRIDE confirm findings from the primary analysis and preliminarily suggest that the advantages of once-monthly PP in effectiveness apply across all antipsychotic classes (ie, between once-monthly PP and conventional oral antipsychotics and between oncemonthly PP and atypical oral antipsychotics). As expected, differential AE profiles were observed between the conventional oral antipsychotics, atypical oral antipsychotics, and once-monthly PP groups. Given the varied safety profiles among these agents, pooled data and the deselection process could have masked tolerability issues associated with any one oral agent. These findings provide insight into the differential effectiveness and side-effect profiles of the once-monthly PP versus daily oral conventional antipsychotics and daily oral atypical antipsychotics; however, larger groups of patients are required to draw more definitive conclusions.

\section{Disclosures}

E. Kim, H. L. Starr, and L. Alphs are employees of Janssen Scientific Affairs, LLC, and are Johnson \& Johnson stockholders. L. Mao is an employee of Janssen Research and Development, LLC, and is a Johnson \& Johnson stockholder. C. U. Correll has been a consultant and/or advisor to or has received honoraria from AbbVie, Acadia, Actavis, Alkermes, Eli Lilly, Forum, Genentech, Gerson Lehrman Group, IntraCellular Therapies, Janssen/J\&J, Lundbeck, MedAvante, Medscape, Otsuka, Pfizer, ProPhase, Reviva, Roche, Sunovion, Supernus, Takeda, and Teva. He has received grant support from Bristol-Myers Squibb, Otsuka, Lundbeck, and Takeda. C. U. Correll reports personal fees from Janssen/J\&J, AbbVie, Acadia, Actavis, Alkermes, Eli Lilly, Genentech, Gerson Lehrman Group, IntraCellular Therapies, Lundbeck, MedAvante, Medscape, Otsuka, Pfizer, ProPhase, Reviva, Roche, Sunovion, Supernus, Takeda, and Teva, outside the submitted work. 


\section{Supplementary material}

For supplementary material/s referred to in this article, please visit http://dx.doi.org/10.1017/S109285291600 0444

\section{REFERENCES:}

1. National Institute of Mental Health. Schizophrenia [Web page]. Washington, DC: National Institute of Mental Health; 2014. http:// www.nimh.nih.gov/statistics/1SCHIZ.shtml. Accessed May 1, 2014.

2. van Os J, Kapur S. Schizophrenia. Lancet. 2009; 374(9690): 635-645.

3. Pandina GJ, Lindenmayer JP, Lull J, et al. A randomized, placebocontrolled study to assess the efficacy and safety of 3 doses of paliperidone palmitate in adults with acutely exacerbated schizophrenia. J Clin Psychopharmacol. 2010; 30(3): 235-244.

4. Pandina G, Lane R, Gopal S, et al. A double-blind study of paliperidone palmitate and risperidone long-acting injectable in adults with schizophrenia. Prog Neuropsychopharmacol Biol Psychiatry. 2011; 35(1): 218-226.

5. Nasrallah HA. The case for long-acting antipsychotic agents in the post-CATIE era. Acta Psychiatr Scand. 2007; 115(4): 260-267.

6. Chue P, Eerdekens M, Augustyns I, et al. Comparative efficacy and safety of long-acting risperidone and risperidone oral tablets. Eur Neuropsychopharmacol. 2005; 15(1): 111-117.

7. Bai YM, Chen TT, Wu B, et al. A comparative efficacy and safety study of long-acting risperidone injection and risperidone oral tablets among hospitalized patients: 12-week randomized, singleblind study. Pharmacopsychiatry. 2006; 39(4): 135-141.

8. Macfadden W, Ma YW, Thomas HJ, Bossie CA, Alphs L. A prospective study comparing the long-term effectiveness of injectable risperidone long-acting therapy and oral aripiprazole in patients with schizophrenia. Psychiatry. 2010; 7(11): 23-31.

9. Kishimoto T, Robenzadeh A, Leucht C, et al. Long-acting injectable vs oral antipsychotics for relapse prevention in schizophrenia: a metaanalysis of randomized trials. Schizophr Bull. 2014; 40(1): 192-213.

10. Janssen Pharmaceuticals, Inc. INVEGAR SUSTENNAR (paliperidone palmitate) extended-release injectable suspension, for intramuscular use. 2012. Titusville, NJ: Janssen Pharmaceuticals, Inc.

11. Hargarter L, Cherubin P, Bergmans $P$, et al. Intramuscular longacting paliperidone palmitate in acute patients with schizophrenia unsuccessfully treated with oral antipsychotics. Prog Neuropsychopharmacol Biol Psychiatry. 2015; 58: 1-7.

12. Fu DJ, Bossie CA, Kern Sliwa J, Ma YW, Alphs L. Paliperidone palmitate versus risperidone long-acting injection in markedly-to-severely ill schizophrenia subjects: onset of efficacy with recommended initiation regimens. Clin Schizophr Relat Psychoses. 2014; 8(2): 101-109, 109A.

13. Hough D, Gopal S, Vijapurkar U, Lim P, Morozova M, Eerdekens M. Paliperidone palmitate maintenance treatment in delaying the time-torelapse in patients with schizophrenia: a randomized, double-blind, placebo-controlled study. Schizophr Res. 2010; 116(2-3): 107-117.

14. Nasrallah HA, Gopal S, Gassmann-Mayer C, et al. A controlled, evidence-based trial of paliperidone palmitate, a long-acting injectable antipsychotic, in schizophrenia. Neuropsychopharmacology. 2010; 35(10): 2072-2082.

15. Alphs L, Bossie CA, Sliwa JK, Ma YW, Turner N. Onset of efficacy with acute long-acting injectable paliperidone palmitate treatment in markedly to severely ill patients with schizophrenia: post hoc analysis of a randomized, double-blind clinical trial. Ann Gen Psychiatry. 2011; 10(1): 12.

16. Bossie CA, Sliwa JK, Ma YW, Fu DJ, Alphs L. Onset of efficacy and tolerability following the initiation dosing of long-acting paliperidone palmitate: post-hoc analyses of a randomized, doubleblind clinical trial. BMC Psychiatry. 2011; 11 : 79.

17. Bossie CA, Fu DJ, Sliwa JK, Ma YW, Alphs L. Tolerability of initiation doses of once-monthly paliperidone palmitate in patients with recently diagnosed schizophrenia in an acute treatment trial. Ther Adv Psychopharmacol. 2011; 1(4): 111-124.

18. González-Rodríguez A, Catalán R, Penadés R, et al. Profile of paliperidone palmitate once-monthly long-acting injectable in the management of schizophrenia: long-term safety, efficacy, and patient acceptability-a review. Patient Prefer Adherence. 2015; 9: 695-706.

19. Li H, Rui Q, Ning X, Xu H, Gu N. A comparative study of paliperidone palmitate and risperidone long-acting injectable therapy in schizophrenia. Prog Neuropsychopharmacol Biol Psychiatry. 2011; 35(4): 1002-1008.

20. Gopal S, Xu H, Bossie C, et al. Incidence of tardive dyskinesia: a comparison of long-acting injectable and oral paliperidone clinical trial databases. Int J Clin Pract. 2014; 68(12): 1514-1522.

21. Schreiner A, Bergmans $\mathrm{P}$, Cherubin $\mathrm{P}$, et al. A prospective flexible-dose study of paliperidone palmitate in nonacute but symptomatic patients with schizophrenia previously unsuccessfully treated with oral antipsychotic agents. Clin Ther. 2014; 36(10): 1372-1388.

22. Alphs L, Bossie CA, Sliwa JK, Fu DJ, Ma YW, Hulihan J. Paliperidone palmitate and risperidone long-acting injectable in subjects with schizophrenia recently treated with oral risperidone or other oral antipsychotics. Neuropsychiatr Dis Treat. 2013; 9: 341-350.

23. Hough D, Lindenmayer JP, Gopal S, et al. Safety and tolerability of deltoid and gluteal injections of paliperidone palmitate in schizophrenia. Prog Neuropsychopharmacol Biol Psychiatry. 2009; 33(6): 1022-1031.

24. Coppola D, Liu Y, Gopal S, et al. A one-year prospective study of the safety, tolerability and pharmacokinetics of the highest available dose of paliperidone palmitate in patients with schizophrenia. $B M C$ Psychiatry. 2012; 12: 26.

25. Sliwa JK, Bossie CA, Fu DJ, Turkoz I, Alphs L. Long-term tolerability of once-monthly injectable paliperidone palmitate in subjects with recently diagnosed schizophrenia. Neuropsychiatr Dis Treat. 2012; 8: 375-385.

26. Fleischhacker WW, Gopal S, Lane R, et al. A randomized trial of paliperidone palmitate and risperidone long-acting injectable in schizophrenia. Int J Neuropsychopharmacol. 2012; 15(1): 107-118.

27. Alphs L, Mao L, Rodriguez SC, Hulihan J, Starr L. Design and rationale of the paliperidone palimate research in demonstrating effectiveness (PRIDE) study: a novel comparative trial of oncemonthly paliperidone palmitate versus daily oral antipsychotic treatment for delaying time to treatment failure in persons with schizophrenia. J Clin Psychiatry. 2014; 75(12): 1388-1393.

28. Zhang F, Si T, Chiou CF, et al. Efficacy, safety, and impact on hospitalizations of paliperidone palmitate in recent-onset schizophrenia. Neuropsychiatr Dis Treat. 2015; 11: 657-668.

29. Alphs L, Benson C, Cheshire-Kenny K, Lindenmayer JP, Mao L, Rodriguez SC. Real-world outcomes of -paliperidone palmitate compared to daily oral antipsychotic therapy in schizophrenia: a randomized, open-label, review board-blinded 15-month study. J Clin Psychiatry. 2015; 76(5): 554-561.

30. American Psychiatric Association. Diagnostic and Statistical Manual of Mental Disorders, 4th ed. Washington, DC: American Psychiatric Association; 1994.

31. Sheehan DV, Lecrubier Y, Harnett Sheehan K, et al. The MiniInternational Neuropsychiatric Interview (M.I.N.I.): the development and validation of a structured diagnostic psychiatric interview for DSM-IV and ICD-10. J Clin Psychiatry. 1998; 59(Suppl 20): 22-33.

32. Lavori PW, Rush AJ, Wisniewski SR, et al. Strengthening clinical effectiveness trials: equipoise-stratified randomization. Biol Psychiatry. 2001; 50(10): 792-801. 
33. Chue P, Chue J. A review of paliperidone palmitate. Expert Rev Neurother. 2012; 12(12): 1383-1397.

34. Kane JM, Kishimoto T, Correll CU. Non-adherence to medication in patients with psychotic disorders: epidemiology, contributing factors and management strategies. World Psychiatry. 2013; 12(3): 216-226.

35. Peuskens J, Pani L, Detraux J, De HM. The effects of novel and newly approved antipsychotics on serum prolactin levels: a comprehensive review. CNS Drugs. 2014; 28(5): 421-453.

36. Leucht S, Cipriani A, Spineli L, et al. Comparative efficacy and tolerability of 15 antipsychotic drugs in schizophrenia: a multipletreatments meta-analysis. Lancet. 2013; 382(9896): 951-962.
37. Citrome L. New second-generation long-acting injectable antipsychotics for the treatment of schizophrenia. Expert Rev Neurother. 2013; 13(7): 767-783.

38. Schreiner A, Aadamsoo K, Altamura AC, et al. Paliperidone palmitate versus oral antipsychotics in recently diagnosed schizophrenia. Schizophr Res. 2015; 169(1-3): 393-399.

39. Markowitz M, Fu DJ, Levitan B, Gopal S, Turkoz I, Alphs L. Long-acting injectable paliperidone palmitate versus oral paliperidone extended release: a comparative analysis from two placebo-controlled relapse prevention studies. Ann Gen Psychiatry. $2013 ; 12(1): 22$. 